\title{
A statistical fractal-diffusive avalanche model of a slowly-driven self-organized criticality system ${ }^{\star}$
}

\author{
M. J. Aschwanden \\ Lockheed Martin Advanced Technology Center, Solar \& Astrophysics Laboratory, Org. ADBS, Bldg. 252, 3251 Hanover St., \\ Palo Alto, CA 94304, USA \\ e-mail: aschwanden@lmsal.com
}

Received 10 October 2011 / Accepted 12 December 2011

\begin{abstract}
Aims. We develop a statistical analytical model that predicts the occurrence frequency distributions and parameter correlations of avalanches in nonlinear dissipative systems in the state of a slowly-driven self-organized criticality (SOC) system.

Methods. This model, called the fractal-diffusive SOC model, is based on the following four assumptions: (i) the avalanche size $L$ grows as a diffusive random walk with time $T$, following $L \propto T^{1 / 2}$; (ii) the energy dissipation rate $f(t)$ occupies a fractal volume with dimension $D_{S}$; (iii) the mean fractal dimension of avalanches in Euclidean space $S=1,2,3$ is $D_{S} \approx(1+S) / 2$; and (iv) the occurrence frequency distributions $N(x) \propto x^{-\alpha_{x}}$ based on spatially uniform probabilities in a SOC system are given by $N(L) \propto L^{-S}$, with $S$ being the Eudlidean dimension. We perform cellular automaton simulations in three dimensions $(S=1,2,3)$ to test the theoretical model. Results. The analytical model predicts the following statistical correlations: $F \propto L^{D_{S}} \propto T^{D_{S} / 2}$ for the flux, $P \propto L^{S} \propto T^{S / 2}$ for the peak energy dissipation rate, and $E \propto F T \propto T^{1+D_{S} / 2}$ for the total dissipated energy; the model predicts powerlaw distributions for all parameters, with the slopes $\alpha_{T}=(1+S) / 2, \alpha_{F}=1+(S-1) / D_{S}, \alpha_{P}=2-1 / S$, and $\alpha_{E}=1+(S-1) /\left(D_{S}+2\right)$. The cellular automaton simulations reproduce the predicted fractal dimensions, occurrence frequency distributions, and correlations within a satisfactory agreement within $\approx 10 \%$ in all three dimensions.

Conclusions. One profound prediction of this universal SOC model is that the energy distribution has a powerlaw slope in the range of $\alpha_{E}=1.40-1.67$, and the peak energy distribution has a slope of $\alpha_{P}=1.67$ (for any fractal dimension $D_{S}=1, \ldots, 3$ in Euclidean space $S=3$ ), and thus predicts that the bulk energy is always contained in the largest events, which rules out significant nanoflare heating in the case of solar flares.
\end{abstract}

Key words. Sun: flares - methods: statistical - instabilities

\section{Introduction}

The statistics of nonlinear processes in the universe often shows powerlaw-like distributions, most conspicously in energetic dynamic phenomena in astrophysics (e.g., solar and stellar flares, pulsar glitches, auroral substorms) and in catastrophic events in geophysics (e.g., earthquakes, landslides, or forest fires). The most widely known example is the distribution of earthquake magnitudes, which has a powerlaw slope of $\alpha \approx 2.0$ for the differential frequency distribution (Turcotte 1999), the socalled Gutenberg-Richter (1954) law. Bak et al. (1987, 1988) introduced the theoretical concept of self-organized criticality (SOC), which has been initially applied to sandpile avalanches at a critical angle of repose, and has been generalized to nonlinear dissipative systems that are driven in a critical state. Comprehensive reviews on this subject can be found for applications in geophysics (Turcotte 1999), solar physics (Charbonneau et al. 2001), and astrophysics (Aschwanden 2011).

Hallmarks of SOC systems are the scale-free powerlaw distributions of various event parameters, such as the peak energy dissipation rate $P$, the total energy $E$, or the time duration $T$ of events. While the powerlaw shape of the distribution function can be explained by the statistics of nonlinear processes that have an exponential growth phase and saturate after a random time

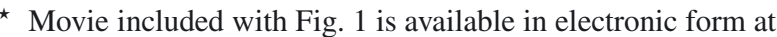
http://www. aanda.org
}

interval (e.g., Willis \& Yule 1922; Fermi 1949; Rosner \& Vaiana 1978; Aschwanden et al. 1998; Aschwanden 2004, 2011), no general theoretical model has been developed that predicts the numerical value of the powerlaw slope of SOC parameter distributions. Simple analytical models that characterize the nonlinear growth phase with an exponential growth time $\tau_{G}$ and the random distribution of risetimes with an average value of $t_{S}$, predict a powerlaw slope of $\alpha_{P}=1+t_{S} / \tau_{G}$ for the energy dissipation rate (e.g., Rosner \& Vaiana 1978; Aschwanden et al. 1998), but cellular automaton simulations suggest a much more intermittent energy release process than the idealized case of an avalanche with a single growth and decay phase. An alternative theoretical explanation for a slope $\alpha_{E}=3 / 2$ was put forward by a dimensional argument (Litvinenko 1998a), which can be derived from the definition of the kinetic energy of convective flows, but this model entails a specific physical mechanism that has not universal validity for SOC systems.

In this Paper we propose a more general concept where the powerlaw slope of the occurrence frequency distribution of SOC parameters depends on the fractal geometry of the energy dissipation domain. We aim for a universal statistical model of nonlinear energy dissipation processes that is independent of any particular physical mechanism. The fractal structure of selforganized processes has been stressed prominently from beginning (Bak et al. 1987, 1988; Bak \& Chen 1989), but no quantitative theory has been put forward that links the fractal geometry to the size distribution of SOC events. Fractals have been studied 
independently (e.g., Mandelbrot 1977, 1983, 1985), while the fractal geometry of SOC avalanches was postulated (e.g., see textbooks of Bak 1996; Sornette 2004; Aschwanden 2011), but no general self-consistent model has been attempted.

This paper presents an analytical theory that derives a theoretical framework to quantitatively link the concept of fractal dimensions to the occurrence frequency distributions of SOC avalanche events (Sect. 2), tests of the analytical theory with numerical simulations of cellular automaton models in three Euclidean dimensions (Sect. 3), a comparison and application to solar flares (Sect. 4), and a summary of the model assumptions and conclusions (Sect. 5).

\section{Theory}

We derive in this section a general model of the statistics of SOC processes, but make use of a specific example of a SOC avalanche that is numerically simulated with a cellular automaton code and described in more detail in Sect. 3, to illustrate and validate our theoretical derivation.

\subsection{Diffusive random walk in cellular automaton avalanches}

An avalanche in a cellular automaton model propagates via nearest-neighbor interactions in random directions, wherever an unstable node is found. The state of self-organized criticality ensures that the entire system is close to the instability threshold, and thus every direction of instability propagation is equally likely once a starting location is triggered (if the re-distribution rule is defined to be isotropic). We can therefore model the propagation of unstable nodes with a random walk in a S-dimensional space, which has the characteristics of a diffusion process and propagates in the statistical average a distance $x(t)$ that depends on the time $t$ as,

$x(t) \propto t^{1 / 2}$.

As a plausibility test we check this first assumption with an example of a numerical simulation of a cellular automaton model that is described in more detail in Sect. 3. The simulated avalanche shown in Fig. 1 lasts for a duration of 712 time steps and we show snapshots of the energy dissipation rate $\mathrm{d} e / \mathrm{d} t$ in Fig. 1. The complete time evolution of the energy dissipation rate $\mathrm{d} e(t) / \mathrm{d} t$, the total energy $e(t)$, the fractal dimension $D_{2}(t)$, and radius of the avalanche area $r(t)$ as a function of time is shown in Fig. 3. A movie of the avalanche that shows the time evolution for all 712 time steps is included in the electronic supplementary data of this journal. The movie illustrates also that the instantaneous propagation direction of the avalanche is nearly isotropic, as we assumed here, regardless of the prior evolution in the neighborhood of the instantaneous energy release. Apparently, many of the grid points that have already been touched by the avalanche previously are still in a meta-stable state near the critical threshold that enables next-neighbor interactions.

We calculate the total time-integrated area $a(t)$ of the avalanche by summing up all unstable nodes where energy dissipation happened during the time interval $[0, t]$ (counting each unstable node only once, even when the same node was unstable more than once), and measure the mean radius $r(t)$ of the avalanche area by

$$
r(t)=\sqrt{\frac{a(t)}{\pi}},
$$

which closely follows the diffusive random walk distance $x(t) \propto$ $t^{1 / 2}$, as it can be seen in Fig. 3 (bottom right panel), or from the circular area with radius $r(t)$ drawn around the starting point of the avalanche in Fig. 1 (dashed circles around center marked with a cross). Thus, the total time-integrated area $a(t)$ of an avalanche increases with a diffusive scaling. If we define $T$ to be the total time duration of the avalanche, and $a_{T}=a(t=T)=$ $\pi r^{2}(t=T)$ the final area of the avalanche, then the final linear size $L$ of the time-integrated avalanche is,

$L=\sqrt{a_{T}}=\sqrt{\pi} r(t=T)$.

According to the diffusive propagation we expect then not only a time evolution $r(t) \propto t^{1 / 2}$ (Eq. (1)) for individual avalanches (in the statistical average), but also a statistical relationship between the size scales $L$ and the time durations $T$ for an ensemble of many avalanches,

$L \propto T^{1 / 2}$.

The time-integrated area $a_{T}$ of an avalanche, as the outlined contours in Fig. 1 show, appears to be a contiguous, space-filling area that is essentially non-fractal, although it has some ragged boundaries. For an example of a 3-dimensional avalanche see McIntosh et al. (2002), which also shows essentially a spacefilling 3-D topology for the time-integrated avalanche volume. This conclusion is somewhat intuitive in cellular automaton models, where avalanches can propagate over nearest neighbors only, and thus will tend to cover a near space-filling area for isotropic propagation. Although the boundaries are somewhat ragged, the area $A$ is equivalent to a circular area with a mean radius of $r(t)$, and thus the fractal dimension would be approximately $D=2$, if a box-counting method is applied within a quadratic area with size $a_{T}=L^{2}=\pi r^{2}$. Thus, we define that the total avalanche area has a Euclidean space-filling topology within the diffusive boundary, and can be characterized with a size scale $L$ and area $a_{T}=L^{2}$. Generalizing to 3-D space, the volume $v$ of the avalanche boundary can be characterized by $v_{T}=L^{3}$.

\subsection{The fractal geometry of instantaneous energy dissipation}

While we established the space-filling nature of the timeintegrated avalanche area with a (non-fractal) Euclidean dimension in the foregoing section, we will now, in contrast, derive the theorem that the instantaneous area of energy dissipation in avalanches is fractal. It is actually a key concept of SOC systems that the spatial structure of avalanches is fractal. Bak \& Chen (1989) express this most succintly in their abstract: "Fractals in nature originate from self-organized critical dynamical processes".

As it can be seen from the snapshots of an evolving avalanche shown in Fig. 1, the instantaneous areas of energy dissipation cover a fraction of the solid area $a(t)$ that is encompassed by the diffusive boundary. A detailed inspection of the shapshots shown in Fig. 1 even reveals a checkerboard pattern of instantaneous avalanche maps that emphasizes the fractal topology of cellular automaton avalanches. We make now the second major assumption that the area $A(t)$ of instantaneous energy dissipation $\mathrm{d} e(t) / \mathrm{d} t$ is fractal, or that the volume $V(t)$ for $3-\mathrm{D}$ avalanches is fractal, respectively. To simplify the nomenclature, we will generally refer to the fractal volume $V_{S}$ in S-dimensional Euclidean space, which corresponds to $V_{3}=V$ for fractal volumes in 3-D space, to $V_{2}=A$ for fractal areas 
M. J. Aschwanden: A statistical fractal-diffusive avalanche model of a slowly-driven self-organized criticality system
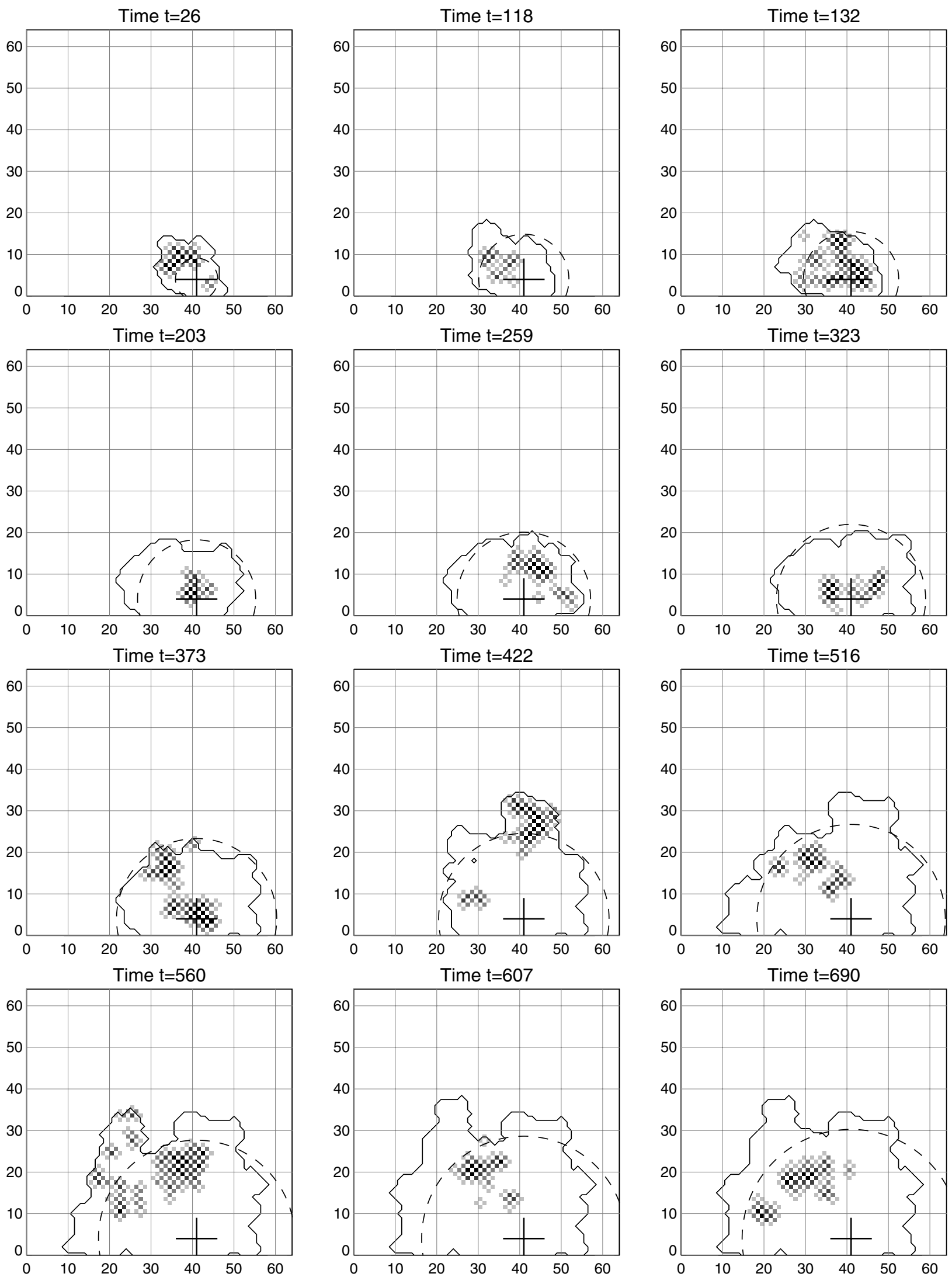

Fig. 1. Time evolution of the largest avalanche event \#1628 in the 2-D cellular automaton simulation with grid size $N=64^{2}$. The 12 panels show snapshots at particular burst times from $t=26$ to $t=690$ when the energy dissipation rate peaked. Active nodes where energy dissipation occurs at time $t$ are visualized with black and grey points, depending on the energy dissipation level. The starting point of the avalanche occurred at pixel $(x, y)=(41,4)$, which is marked with a cross. The time-integrated envelop of the avalanche is indicated with a solid contour, and the diffusive avalanche radius $r(t)=t^{1 / 2}$ is indicated with a dashed circle. The temporal evolution is shown in a movie available in the on-line version. 
in 2-D space, and $V_{1}=X$ for fractal lengths in 1-D space. (Note that we use uppercase symbols $V, A, X$ for fractal parameters, while we use lowercase symbols $v, a, x$ for non-fractal Euclidean parameters.) A fractal volume $V_{S}$ can be defined by the Hausdorff dimension $D_{S}$ in $S$-dimensional Euclidean space,

$D_{S}=\lim _{x \mapsto 0} \frac{\log V_{S}}{\log x}$

where $V_{S}$ is the fractal volume with Euclidean scale $x$, or by the scaling law,

$V_{S} \propto x^{D_{S}}$.

In Fig. 2 we demonstrate the fractal nature of the 12 instantaneous avalanche snapshots shown in Fig. 1. We rebin the avalanche area into macropixels with sizes of $\Delta x_{i}=2^{i}, i=$ $0, \ldots, 3$ (or $x=1,2,4,8$ ), measure the number of macropixels $A_{i}$ that cover the instantaneous avalanche area, use the diffusive scaling $x(t) \propto t^{1 / 2}$ (Eq. (1)) for the rebinned length units $x_{i}=x(t) / \Delta x_{i}$, and determine the Hausdorff dimemsion $D_{2}$ from the linear regression fit $\log \left(A_{i}\right)=D_{2} \log \left(x_{i}\right)$. We find that that the 4 datapoints for each of the 12 cases exhibit a linear relationship, which proves the fractality of the avalanche areas. The average fractal dimension of the 12 timesteps shown in Figs. 1 and 2 is $D_{2}=1.43 \pm 0.17$.

We measure now the fractal dimension $D_{2}$ of the instantaneous energy dissipation volume in the 2-D avalanche for all 700 time steps of its duration, shown for 12 time instants in Fig. 1. The unstable nodes (signifying instantaneous energy dissipation) are counted in each time step, which yield a number for the fractal volume or area $A(t)=V_{2}(t)$, while the size $x$ of the encompassing box is determined from the area of the timeintegrated avalanche, i.e., $x(t)=\sqrt{a(t)}$, which yields the time evolution of the fractal dimension $D_{2}(t)=\log \left[V_{2}(t)\right] / \log [x(t)]$ (Eq. (5)) as a function of time, shown in the top right panel in Fig. 3. The fractal dimension $D_{2}(t)$ fluctuates around a constant mean value of $D_{2}=1.45 \pm 0.13$, which is close to the arithmetic mean of the minimum dimension $D_{2, \min } \approx 1$ and maximum Euclidean limit $D_{2, \max }=2$, i.e. $\left\langle D_{2}\right\rangle \approx\left(D_{2, \min }+D_{2, \max }\right) / 2=$ $3 / 2$. This corroborates our second major assumption that the instantaneous volume of energy dissipation is fractal, and that the fractal dimension can be approximated by a mean (timeindependent and size-independent) constant during the evolution of avalanches, in the statistical average.

If we moreover define a mean energy dissipation rate quantum $\langle\Delta E\rangle$ per unstable node, which is indeed almost a constant for a cellular automaton model near the critical state, we expect a scaling of the instantaneous dissipation rate (or flux) $f(t)$ that is proportional to the instantaneous dissipation volume $V_{S}$ (with Eq. (6)),

$f(t)=\frac{\mathrm{d} e(t)}{\mathrm{d} t} \propto\langle\Delta E\rangle V_{S}(t)=\langle\Delta E\rangle x(t)^{D_{S}}$.

Combining this with the diffusive expansion of the boundary $x(t) \propto t^{1 / 2}$ (Eq. (1)), we can then predict the average time evolution of the energy dissipation rate $f(t)=\mathrm{d} e(t) / \mathrm{d} t$,

$f(t)=\frac{\mathrm{d} e(t)}{\mathrm{d} t} \propto\langle\Delta E\rangle t^{\left(D_{S} / 2\right)}$

Integrating Eq. (8) in time, we obtain the time evolution of the total dissipated energy, $e(t)$,

$e(t)=\int_{0}^{t} \frac{\mathrm{d} e(\tau)}{\mathrm{d} \tau} \mathrm{d} \tau \propto \int_{0}^{t} \tau^{D_{S} / 2} \mathrm{~d} \tau=t^{\left(1+D_{S} / 2\right)}$.

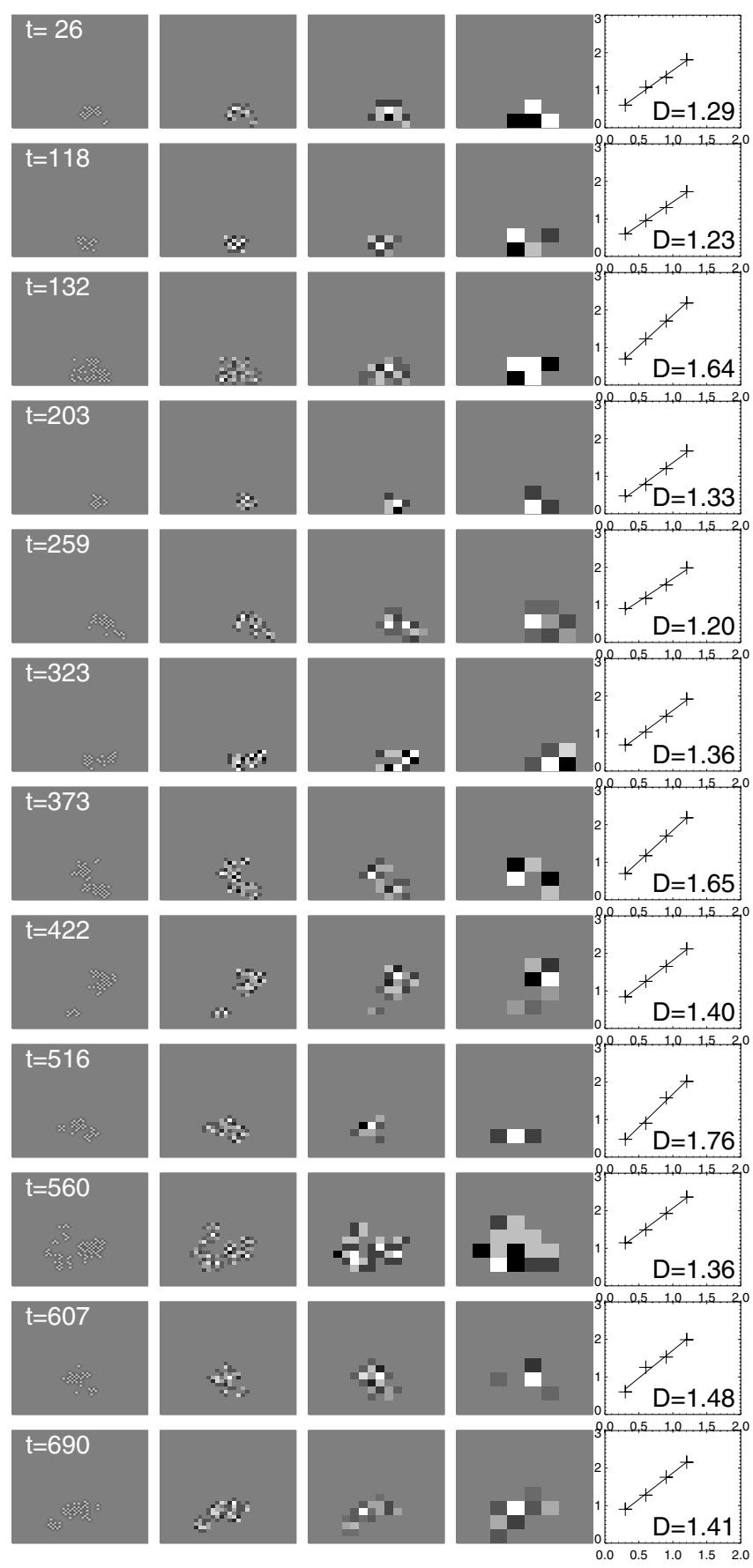

Fig. 2. Determination of the fractal dimension $D_{2}=\log A_{i} / \log x_{i}$ for the instantaneous avalanche sizes of the 12 time steps of the avalanche event shown in Fig. 1. Each row is a different time step and each column represents a different binning of macropixels $\left(\Delta x_{i}=1,2,4,8\right)$. The fractal dimension is determined by a linear regression fit shown on the right-hand side. The mean fractal dimension of the 12 avalanche snapshots is $D_{2}=1.43 \pm 0.17$.

Hence, for our 2-D avalanche (with $D_{2}=3 / 2$ ) we expect an evolution of $e(t) \propto t^{(7 / 4)}$, which indeed closely matches the actually simulated cellular automaton case, as we see in Fig. 3 (bottom left panel). The time evolution of the energy dissipation rate is shown in Fig. 3 (top left panel), which fluctuates strongly during the entire avalanche, but follows in the statistical average the predicted evolution $\mathrm{d} e(t) / \mathrm{d} t \propto t^{D_{2} / 2}=t^{(3 / 4)}$ for $D_{2} \approx 3 / 2$. Note that our analytical expressions of the time evolution of avalanches, such as the linear size $x(t)$ (Eq. (1)), the instantaneous energy 

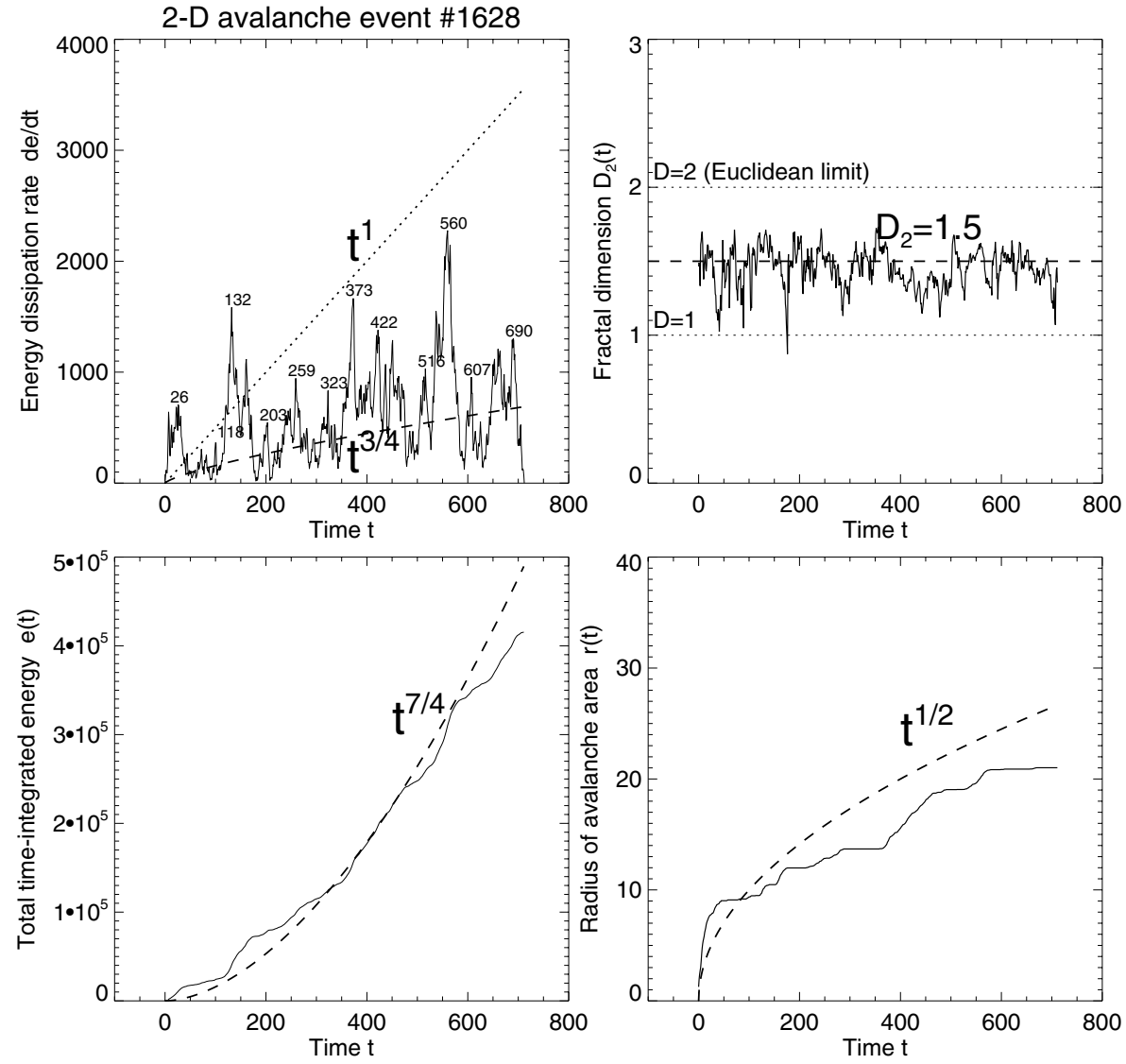

Fig. 3. Time evolution of the largest avalanche event \#1628 in the 2-D cellular automaton simulation with grid size $N=64^{2}$. The time profiles include the instantaneous energy dissipation rate $f(t)=\mathrm{d} e / \mathrm{d} t$ (top left), the time-integrated total energy $e(t)$ (bottom left), the instantaneous fractal dimension $D_{2}(t)$ (top right), and the radius of the avalanche area $r(t)$ (bottom right). The observed time profiles from the simulations are outlined in solid linestyle and the theoretically predicted average evolution in dashed linestyle. The statistically predicted values of the instantaneous energy dissipation rate $f(t) \propto t^{3 / 4}$ (dotted curve) and peak energy dissipation rate $p(t) \propto t^{1}$ (dashed curve) after a time interval $t$ are also shown (top left panel). The 12 time labels from 26 to 690 (top left frame) correspond to the snapshot times shown in Fig. 1. dissipation rate $f(t)$ (Eqs. (7), (8)), or the total dissipated energy $e(t)$ (Eq. (9)), do not predict the specific evolution of a single avalanche event, but rather the statistical expectation value of a large ensemble of avalanches, similar to the statistical nature of the diffusive random walk model (Eq. (1)).

The time evolution of the instantaneous energy dissipation rate $f(t)$ fluctuates strongly, as it can be seen in for the largest avalanche simulated in a cellular automaton model (Fig. 3, top left panel). We might estimate the peak values that can be obtained statistically (after a time duration $t$ ) from the optimum conditions when the fractal filling factor of the avalanche reaches a near-Euclidean filling, i.e., in the limit of $D_{S} \mapsto S$. Replacing the fractal dimension $D_{S}$ by the Euclidean limit $S$ in Eqs. (7) and (8) yields then (as an upper limit) an expectation value for the peak $p(t)$,

$p(t) \propto\langle\Delta E\rangle V_{S}^{\max }(t) \propto\langle\Delta E\rangle x(t)^{S} \propto\langle\Delta E\rangle t^{(S / 2)}$.

Denoting the energy dissipation rate in the statistical average after time $t=T$ with with $F=f(t=T)$, the peak energy dissipation rate with $P=p(t=T)$, and the total energy of the avalanche with $E=e(t=T)$, it follows from Eqs. (8)-(10) that $E \propto F T=P^{D_{S} / S} T$, and we expect then the following correlations between the three parameters $E, F, P$ and $T$ for an ensemble of avalanches,

$$
\begin{aligned}
& E \propto T^{1+D_{S} / 2} \\
& F \propto T^{D_{S} / 2} \\
& P \propto T^{S / 2} .
\end{aligned}
$$

For instance, for a 2-D avalanche with an average fractal dimension of $D_{2}=3 / 2$ we expect the following two correlations, $E \propto T^{7 / 4}, F \propto T^{3 / 4}$, and $P \propto T^{1}$ (see Fig. 3). The powerlaw indices for the correlated parameters are listed for the three Euclidean dimensions $S=1,2,3$ separately in Table 1 .

\subsection{Occurrence frequency distributions}

Considering the probability of an avalanche with volume $V$, the statistical likelihood simply scales reciprocally to the volume size $V$, if avalanches are equally likely in every space location of a uniform volume $V_{0}$ of a system in a (self-organized) critical state. This is illustrated in Fig. 4. For the 1-D Euclidean space, $n=1$ avalanche can happen with the maximum size $L=L_{0}$ of the system (top left), $n=2$ avalanches with the half size $L=L_{0} / 2$, or $n=4$ avalanches for a quarter size $L=L_{0} / 4$. For the 2-D Euclidean space, the number of possible avalanches that can be fit into the total area $A_{0}$ of the system is $n=1$ for $A=A_{0}$, $n=2^{2}=4$ for $A=A_{0} / 2$, or $n=2^{4}=16$ for $A=A_{0} / 4$ (second row). For the 3-D Euclidean space we have, correspondingly, $n=1$ for $V=V_{0}, n=2^{3}=8$ for cubes of half size $L=L_{0} / 2$, and $n=4^{3}=64$ for quarter-size cubes with $L=L_{0} / 4$ (bottom row). So, generalizing to $S=1,2,3$ dimensions, we can express the probability for an avalanche of size $L$ and volume $V_{S}=L^{S}$ as,

$N(L) \propto V_{S}^{-1} \propto L^{-S}$.

This simple probability argument is based on the assumption that the number or occurrence frequency of avalanches is equally likely throughout the system, so it assumes a homogeneous distribution of critical states across the entire system.

The occurrence frequency distribution of length scales, $N(L) \propto L^{-S}$ (Eq. (12)), serves as a primary distribution function from which all other occurrence frequency distribution functions $N(x)$ can the derived that have a functional relationship to the primary parameter $L$. First we can calculate the occurrence frequency distribution of avalanche time scales, by using the diffusive boundary propagation relationship $L(T) \propto T^{1 / 2}$ (Eq. (4)), 
Table 1. Theoretically predicted occurrence frequency distribution powerlaw slopes $\alpha$ and power indices $\beta$ of parameter correlations predicted for SOC cellular automatons with Euclidean space dimensions $S=1,2,3$.

\begin{tabular}{lcccc}
\hline \hline Parameter & Theory & $S=1$ & $S=2$ & $S=3$ \\
\hline Fractal Dimension: & $D_{S}=(1+S) / 2$ & 1 & $3 / 2$ & 2 \\
Length scale powerlaw slope: & $\alpha_{L}=S$ & 1 & 2 & 3 \\
Duration powerlaw slope: & $\alpha_{T}=(1+S) / 2$ & 1 & $3 / 2$ & 2 \\
Instantaneous energy dissipation rate slope: & $\alpha_{F}=1+(S-1) / D_{S}$ & 1 & $5 / 3$ & 2 \\
Peak energy dissipation rate slope: & $\alpha_{P}=2-1 / S$ & 1 & $3 / 2$ & $5 / 3$ \\
Energy powerlaw slope: & $\alpha_{E}=1+(S-1) /\left(D_{S}+2\right)$ & 1 & $9 / 7$ & $3 / 2$ \\
& & & & \\
Diffusive scaling of length $L$ with duration $T$, & $L \propto T^{1 / 2}$ & $L \propto T^{1 / 2}$ & $L \propto T^{1 / 2}$ & $L \propto T^{1 / 2}$ \\
Correlation of peak rate $F$ with duration $T$, & $F \propto T_{S} / 2$ & $F \propto T^{1 / 2}$ & $F \propto T^{3 / 4}$ & $F \propto T^{1}$ \\
Correlation of peak rate $P$ with duration $T$, & $P \propto T^{S / 2}$ & $P \propto T^{1 / 2}$ & $P \propto T^{1}$ & $P \propto T^{3 / 2}$ \\
Correlation of energy $E$ with duration $T$, & $E \propto T^{1+D_{S} / 2}$ & $E \propto T^{3 / 2}$ & $E \propto T^{7 / 4}$ & $E \propto T^{2}$ \\
\hline
\end{tabular}

by substituting the variable $T$ for $L$ in the distribution $N(L)$ (Eq. (12)),

$N(T) \mathrm{d} T=N(L[T])\left|\frac{\mathrm{d} L}{\mathrm{~d} T}\right| \mathrm{d} T \propto T^{-[(1+S) / 2]} \mathrm{d} T$.

Subsequently we can derive the occurrence frequency distribution function $N(F)$ for the statistically average energy dissipation rate $F=f(t=T)$ using the relationship $F(T) \propto T^{D_{S} / 2}$ (Eq. (11)),

$N(F) \mathrm{d} F=N(T[F])\left|\frac{\mathrm{d} T}{\mathrm{~d} F}\right| \mathrm{d} F \propto F^{-\left[1+(S-1) / D_{S}\right]} \mathrm{d} F$,

the occurrence frequency distribution function of the peak energy dissipation rate $P$ using the relationship relationship $P(T) \propto$ $T^{S / 2}$ (Eq. (11)),

$N(P) \mathrm{d} P=N(T[P])\left|\frac{\mathrm{d} T}{\mathrm{~d} P}\right| \mathrm{d} P \propto P^{-[2-1 / S]} \mathrm{d} P$,

and the occurrence frequency distribution function $N(E)$ for the total energy $E$ using the relationship $E(T) \propto T^{1+D_{S} / 2}$ (Eq. (11)),

$N(E) \mathrm{d} E=N(T[E])\left|\frac{\mathrm{d} T}{\mathrm{~d} E}\right| \mathrm{d} E \propto E^{-\left[1+(S-1) /\left(D_{S}+2\right)\right]} \mathrm{d} E$.

Interestingly, this derivation yields naturally powerlaw functions for all parameters $L, T, F, P$, and $E$, which are the hallmarks of SOC systems. In summary, if we denote the occurrence frequency distributions $N(x)$ of a parameter $x$ with a powerlaw distribution with power index $\alpha_{x}$,

$N(x) \mathrm{d} x \propto x^{-\alpha_{x}} \mathrm{~d} x$,

we have the following powerlaw coefficients $\alpha_{x}$ for the parameters $x=T, F, P$, and $E$,

$\alpha_{T}=(1+S) / 2$
$\alpha_{F}=1+(S-1) / D_{S}$
$\alpha_{P}=2-1 / S$
$\alpha_{E}=1+(S-1) /\left(D_{S}+2\right)$.

For instance, for our 2-D cellular automaton model with $S=2$ and $D_{S}=3 / 2$ we predict powerlaw slopes of $\alpha_{T}=3 / 2=1.5$, $\alpha_{F}=5 / 3 \approx 1.67, \alpha_{P}=3 / 2=1.5$, and $\alpha_{E}=9 / 7 \approx 1.28$. The powerlaw coefficients $\alpha_{x}$ are summarized in Table 1 separately for each Euclidean dimension $S=1,2,3$.

\subsection{Estimating the fractal dimension of cellular automatons}

The dynamics of SOC systems is often simulated on computers with cellular automaton codes, which have a $S$-dimensional lattice of nodes, where a discretized mathematical redistribution rule is applied once a local instability threshold is surpassed (e.g., Bak et al. 1987; Lu \& Hamilton 1991; Charbonneau et al. 2001). Avalanches in such cellular automaton models propagate via nearest-neighbor interactions, which includes $(2 S+1)$ nodes in a $S$-dimensional lattice grid: one element is the unstable node, and there are $2 S$ next neighbors. For instance, a 1-dimensional grid in Euclidian space with dimension $S=1$ has $(2 S+1)=3$ nodes involved in a single nearest-neighbor relaxation step, a 2-dimensional grid $(S=2)$ has $(2 S+1)=5$ nodes, and a 3-dimensional grid $(S=3)$ has $(2 S+1)=7$ nodes (Fig. 5$)$.

How can we estimate the fractal dimension for avalanches in such a lattice-based cellular automaton model? The minimum fractal dimension of a growing 3-D avalanche corresponds to a 1-D linear structure, $D_{3, \min }=1$, because avalanches evolve by iterative propagation from one node to the next-neighbor node, so a contiguous linear path from one node to the next neighbor is about the sparsest spatial structure that still enables avalanche growth in a SOC model. If the linear avalanche path would be discontinuous $\left(D_{S}<1\right)$, the avalanche is likely to die out due to a lack of unstable next neighbors, so $D_{S, \min } \approx 1$ represents practically a lower cutoff, which does not exclude occasional values of $D_{S}<1$ (fractal dust). At the other extreme, when all nodes are close to the instability threshold, an avalanche can affect all next neighbors and grow as a nearly space-filling structure with a maximum fractal dimension of $D_{S, \max }=S$ that equals the Euclidean space dimension $S$. The mean average fractal dimension $\left\langle D_{S}\right\rangle$ can then be estimated from the geometric mean of the minimum $V_{S \text {, min }}$ and maximum fractal volume $V_{S, \max }$, which is equivalent to the arithmetic mean of the minimum $D_{S \text {, min }}$ and maximum fractal dimension $D_{S, \max }$ (Eq. (5)),

$\left\langle D_{S}\right\rangle=\frac{\log \langle V\rangle}{\log x}=\frac{\log \sqrt{V_{S, \text { min }} V_{S, \text { max }}}}{\log x}=\frac{D_{S, \text { min }}+D_{S, \text { max }}}{2}$.

From this we predict the following mean fractal dimensions $\left\langle D_{S}\right\rangle$ in different Euclidean spaces with dimensions $S=1,2,3$,

$\left\langle D_{1}\right\rangle=(1+1) / 2=1$

$\left\langle D_{2}\right\rangle=(1+2) / 2=3 / 2$

$\left\langle D_{3}\right\rangle=(1+3) / 2=2$.

or more generally as a function of the Euclidean space dimension $S$,

$\left\langle D_{S}\right\rangle \approx \frac{(1+S)}{2}$ for $S=1,2,3$. 


\section{1-D Avalanches}

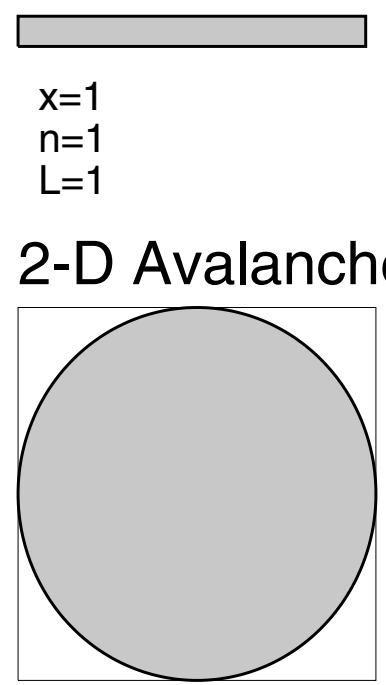

$x=1$
$n=1$
$A=1$

\section{3-D Avalanches}

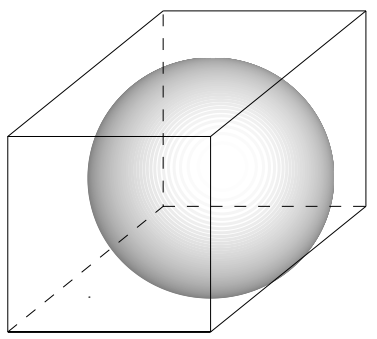

$x=1$

$\mathrm{n}=1$

$\mathrm{V}=1$

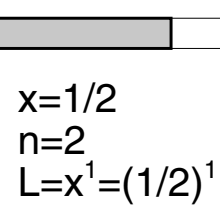

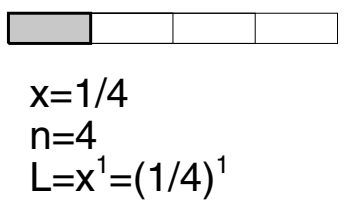




\section{Cellular automaton simulations}

\subsection{Numerical cellular automaton code}

An isotropic cellular automaton model that mimics a SOC system was originally conceived by Bak et al. $(1987,1988)$ and first applied to solar flares by Lu \& Hamilton (1991). A version generalized to $S=1,2,3$ dimensions is given in Charbonneau et al. (2001) and is also summarized in Aschwanden (2011).

The numerical simulations of cellular automaton models take place in a $S$-dimensional cartesian grid, where nodes are localized by discretized coordinates, say with $x_{i j k}$ in $S=3$, and a physical scalar quantity $B_{i j k}=B\left(x=x_{i j k}\right)$ is assigned to each node. The dynamics of the system is initiated by quantized energy inputs $\delta B(t)$ at random locations $x_{i j k}$ with a constant rate as a function of time. At each time step $t$ and spatial node $x_{i j k}$, the local stability is checked by measuring the local $S$-dimensional "curvature" (Charbonneau et al. 2001) with respect to the next neighbor cells (nodes) $x_{n n}=x_{i \pm 1, j \pm 1, k \pm 1}$,

$\Delta B_{i j k}=B_{i j k}-\frac{1}{2 S} \sum B_{n n}$

Most nodes are stable at a particular time if the system is driven slowly, say with an input rate $\delta B /\left\langle B_{i j k}\right\rangle \ll 1$ (Charbonneau et al. 2001). The system is defined to be stable, as long as the local gradients are smaller than some critical threshold value $B_{\mathrm{c}}$. However, once a local gradient exceeds the critical value, i.e., $\Delta B_{i j k} \geq B_{\mathrm{c}}$, a mathematical redistribution rule is applied that smoothes out the local gradient and makes it stable again. The redistribution rule simply spreads the difference $\Delta B_{i j k}$ (or the threshold $B_{\mathrm{c}}$ ) equally to the next neighbors (in an isotropic cellular automaton model),

$$
\boldsymbol{B}_{i j k} \mapsto \boldsymbol{B}_{i j k}-\frac{2 S}{2 S+1} \boldsymbol{B}_{\mathrm{c}}, \quad \boldsymbol{B}_{n n} \mapsto \boldsymbol{B}_{n n}+\frac{1}{2 S+1} \boldsymbol{B}_{\mathrm{c}} .
$$

Note that the amount of the redistributed quantity is the threshold energy $B_{\mathrm{c}}$ in the models of Lu et al. (1993) and Charbonneau et al. (2001), while it is the (larger) amount of the actual gradient $\Delta B_{i j k}$ in the original model of Lu \& Hamilton (1991). If the node is unstable, then the actual gradient is larger than the critical gradient, rather than smaller. This was modified in a later paper ( $\mathrm{Lu}$ et al. 1993) by redistributing the threshold gradient rather than the full gradient, presumably due to numerical instabilities (Liu et al. 2002). This redistribution rule is conservative, in the sense that the quantity $B$ is conserved after every redistribution step, because the same amount is transferred to the next neighbors that is taken away from the central cell. However, although the scalar field quantity $B$ is conserved, the energy $B^{2}$ is not conserved after a redistribution step, because of the nonlinear (quadratic) dependence assumed, which was introduced in analogy to the magnetic field energy density $E_{\text {mag }}=B^{2} / 8 \pi$. In fact, every redistribution of $|\Delta B|>B_{\mathrm{c}}$ dissipates energy from the system, by an amount of (Charbonneau et al. 2001),

$E=\frac{2 S}{2 S+1}\left(\frac{2|\Delta B|}{B_{\mathrm{c}}}-1\right) B_{\mathrm{c}}^{2}$.

Thus, the minimum amount of dissipated energy is for $|\Delta B| \gtrsim B_{\mathrm{c}}$, when the threshold $B_{\mathrm{c}}$ is infinitesimally exceeded by $|\Delta B|$,

$E_{\min }=\frac{2 S}{2 S+1} B_{\mathrm{c}}^{2}$

Once a node $x_{i j k}$ is found to be unstable, the check of unstable cells propagates to the next neighbors $x_{n n}=x_{i \pm 1, j \pm 1, k \pm 1}$ in the next time step and all unstable neighbor cells are subject to the redistribution rule, and progressively continues to the next neighbors each time step until all cells are stable again. Such a chain reaction of next-neighbor redistributions is called an avalanche event (see examples in Fig. 4). Note that a minimum avalanche has to include at least one redistribution step.

In this study the author coded independently such a cellular automaton algorithm according to the specifications given in Sect. 2.5 of Charbonneau et al. (2001) and it was run with exactly the same system parameters, such as the input quantity $\sigma_{1} \leq \delta B \leq \sigma_{2}$ homogeneously distributed in the range of $\sigma_{1}=-0.2$ to $\sigma_{2}=0.8$, the threshold quantity $B_{\mathrm{c}}=5$, with grid sizes of $N=128$ and 256 in one dimension $(S=1), N=32$ and 64 in two dimensions $(S=2)$, and $N=16$ and 24 in three dimensions $(S=3)$. We sampled the total volumes $V$, energies $E$, peak energy dissipation rate $P$, and durations $T$ of avalanches and were able to reproduce the results given in Charbonneau et al. (2001) consistently, although we used different random generators for the input, different time intervals for the initiation phase and onset of SOC $\left(t_{\mathrm{SOC}}=3 \times 10^{6}\right.$ for $N=128$ and $S=1 ; t_{\mathrm{SOC}}=15 \times 10^{6}$ for $N=256$ and $S=1 ; t_{\mathrm{SOC}}=5 \times 10^{6}$ for $N=32$ and $S=2 ; t_{\text {SOC }}=41 \times 10^{6}$ for $N=64$ and $S=2$; $t_{\mathrm{SOC}}=4 \times 10^{6}$ for $N=16$ and $S=3 ; t_{\mathrm{SOC}}=15 \times 10^{6}$ for $N=24$ and $S=3$ ), and slightly different powerlaw fitting procedures. In addition, we calculated also the fractal dimensions of the SOC avalanches from the avalanche volumes $V$ and the size $x$ of the enveloping Euclidean cube $x^{S}$,

$D_{S}=\frac{\log (V)}{\log (x)}$

where $x$ is the largest spatial scale that brackets the fractal avalanche volume in each spatial direction $(x, y, z)$ of the $S$-dimensional Euclidean space. This definition is slightly different from the "radius of gyration" method employed in Charbonneau et al. (2001), but follows more the standard convention of fractal dimensions measured with box-counting methods. In the following we show the results from two runs in each dimension $S=1,2,3$ and compare them with the theoretical predictions made in Sect. 2.

\subsection{Occurrence frequency distributions}

The results of occurrence frequency distributions and correlations are shown for a 1-D (Fig. 6), a 2-D (Fig. 7), and a 3-D cellular automaton code (Fig. 8), and listed in Table 2. We evaluated the powerlaw slopes by a weighted linear regression fit (weighted by the number of avalanche events per bin) by excluding undersampled bins (less than 20 events). Since the exact values depend sometimes on the fitted range, we vary the range of linear regression fits from the full range of the powerlaw part (indicated with black line in Figs. 6-8) to the upper half (indicated with grey line in Figs. 6-8) and calculate the average and standard deviations of the powerlaw slope values.

The values of the predicted and simulated powerlaw slopes are compiled in Table 2 . First of all we notice an extremely good agreement (within a few percents) of the powerlaw slopes $\alpha_{E}$, $\alpha_{P}$, and $\alpha_{T}$ between our simulations and those of Charbonneau et al. (2001) and McIntosh et al. (2002), although we used different codes, initiation times $t_{\mathrm{SOC}}$, and powerlaw fitting procedures. We performed the 1-D to $3-\mathrm{D}$ runs with small cubes $(N=128,32,16)$ as well as with larger cubes $(N=256,64,24)$, which both give very consistent values (Table 2 ). In the following we quote only the values for the larger cubes, which are also shown in Figs. 6-8. 
M. J. Aschwanden: A statistical fractal-diffusive avalanche model of a slowly-driven self-organized criticality system
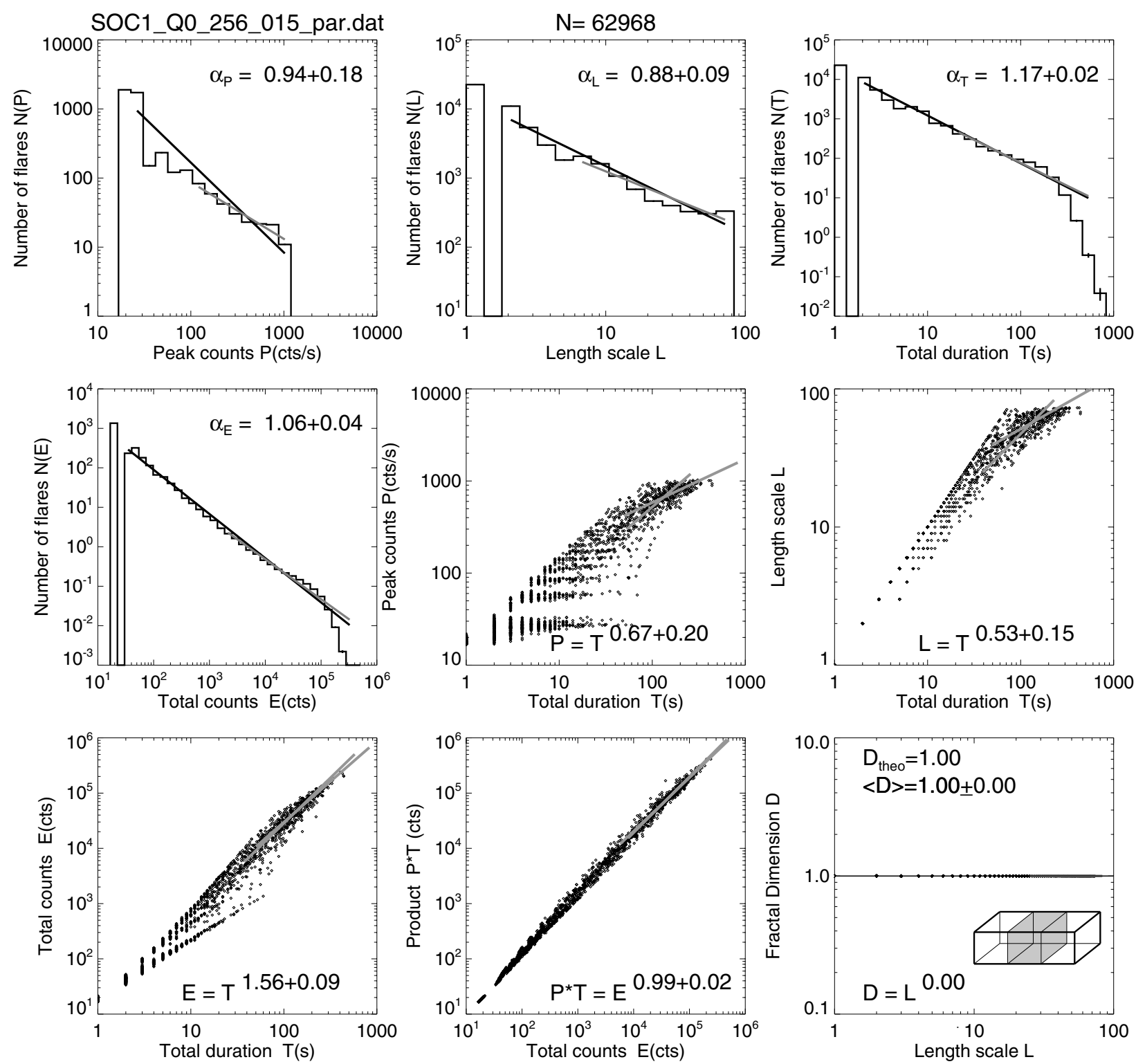

Fig. 6. Cellular automaton simulations with a $N=256$ 1-D lattice produced by a numerical code according to Charbonneau et al. (2001). The frequency distributions of peak energy dissipation rate $P$, total energies $E$, time durations $T$, and fractal avalanche volumes $V$ are shown along with the fitted powerlaw slopes. Correlations between the fractal dimension $D_{1}$ and parameters $E, P$, and $T$ are also shown, fitted in the ranges of $P \geq 50, E \geq 50, T \geq 5$, and $V \geq 2$. Only a representative subset of 2000 events are plotted in the scatterplots.

Considering the agreement between theory and simulations, there is a reasonable agreement for all three space dimensions $S=1,2,3$. For the fractal dimension of avalanches we find $D_{2}=1.60 \pm 0.17$ (predicted $D_{2}=1.5$ ) and $D_{3}=1.94 \pm 0.27$ (predicted $D_{3}=2.0$ ), which are fully consistent with our theoretical estimate of $D_{S}=(1+S) / 2$ (Eq. (21)).

The relationship of the avalanche probability being reciprocal to the volume, $N(L) \propto L^{-S}$ (Eq. (12)) is also approximately confirmed by the simulations, for which we find $\alpha_{L}=0.88 \pm 0.09$ (predicted $\alpha_{L}=1$ for $S=1$ ), $\alpha_{L}=2.14 \pm 0.18$ (predicted $\alpha_{L}=2$ for $S=2$ ), and $\alpha_{L}=2.55 \pm 0.10$ (predicted $\alpha_{L}=3$ for $S=3$ ), where the latter value has the largest error due to the smallest sizes of 3-D cubes with a dynamic range of only about 1 dex. Clearly this relationship agrees better for larger cubes (up to $L=256$ in 1-D simulations). We have to add a caveat that finite-size effects are likely to restrict the size of avalanches at the system boundaries, especially for the 3D cellular automata runs which we run here with a size of $16^{3}$ and $24^{3}$ only. Also uncertainty estimates of the powerlaw slopes are less accurate for small dynamic ranges (i.e., small system size $L$ here) and due to histogram binning. In the real world, however, finite-size effects may also cause modifications of powerlaw distributions, such as the maximum size of active regions or the vertical density scale height of coronal loops.

The total energy powerlaw slope $\alpha_{E}$ (Eq. (18)) fitted from the powerlaw distributions $N(E)$ yields values of $\alpha_{E}=1.06 \pm 0.04$ in 1-D (predicted $\alpha_{E}=1.0$ ), $\alpha_{E}=1.48 \pm 0.03$ in 2-D (predicted $\alpha_{E}=1.28$ ), and $\alpha_{E}=1.50 \pm 0.06$ in 3-D (predicted $\alpha_{E}=1.5$ ), which agree with the predictions within $6 \%, 16 \%$, and $0 \%$.

The peak energy dissipation rate powerlaw slope $\alpha_{P}$ (Eq. (18)) fitted from the powerlaw distributions $N(P)$ yields values of $\alpha_{P}=0.94 \pm 0.18$ in $1-\mathrm{D}\left(\right.$ predicted $\left.\alpha_{P}=1.0\right), \alpha_{P}=$ $1.85 \pm 0.06$ in $2-\mathrm{D}$ (predicted $\alpha_{P}=1.5$ ), and $\alpha_{P}=1.96 \pm 0.14$ in 3-D (predicted $\alpha_{P}=1.67$ ), which agree with the predictions within $6 \%, 23 \%$, and $17 \%$. 

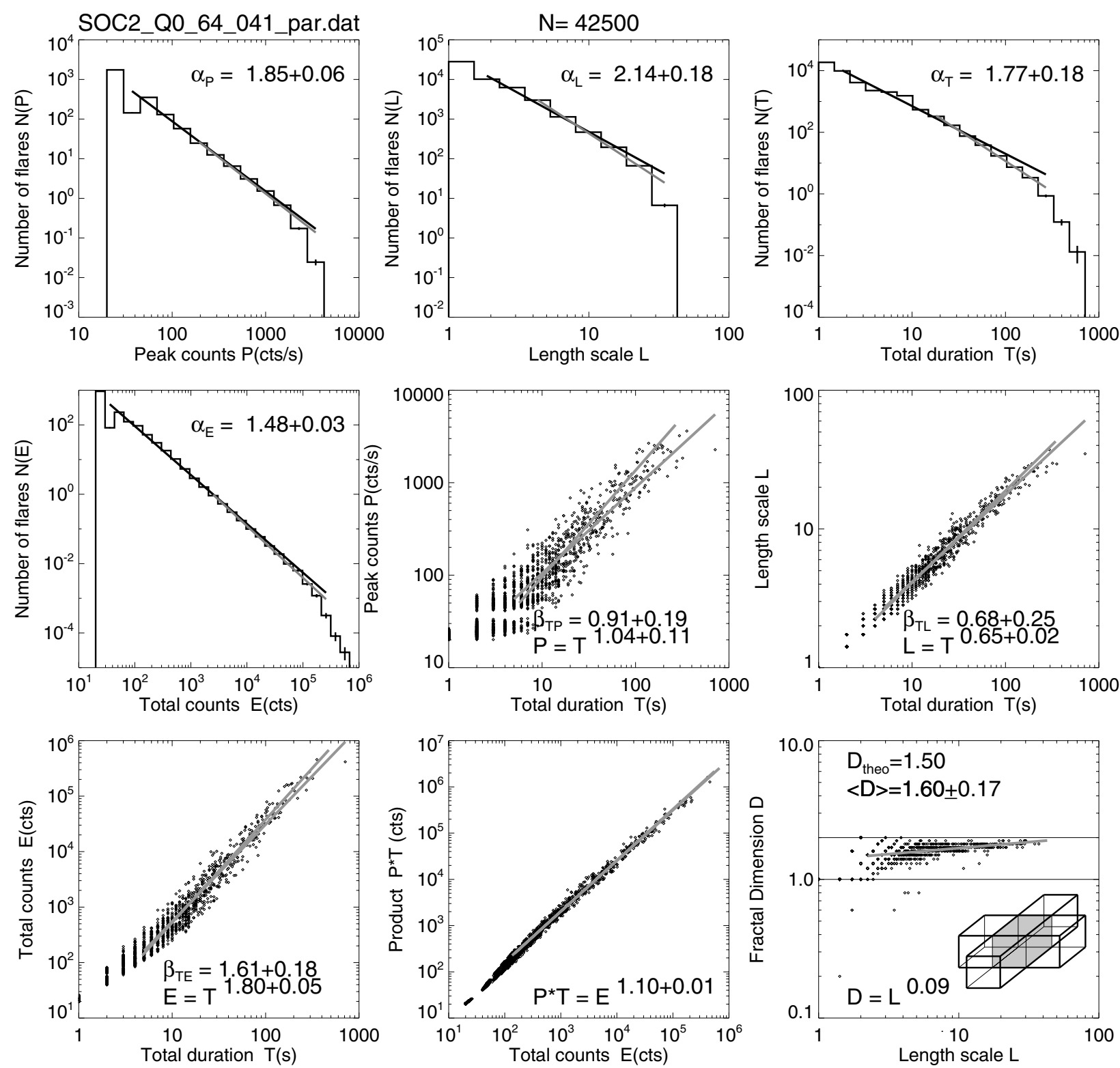

Fig. 7. Cellular automaton simulations with a $N=64^{2}$ 2-D lattice produced by a numerical code according to Charbonneau et al. (2001). Representation otherwise similar to Fig. 6.

The time duration powerlaw slope $\alpha_{T}$ (Eq. (18)) fitted from the powerlaw distributions $N(T)$ yields values of $\alpha_{T}=1.17 \pm$ 0.02 in 1-D (predicted $\alpha_{T}=1.0$ ), $\alpha_{T}=1.77 \pm 0.18$ in 2-D (predicted $\alpha_{T}=1.5$ ), and $\alpha_{T}=1.76 \pm 0.19$ in 3-D (predicted $\alpha_{T}=2.0$ ), which agree with the predictions within $17 \%, 18 \%$, and $12 \%$.

Most occurrence frequency distributions are subject to a drop-off from an ideal powerlaw distribution due to finite-size effects. It is quite satisfactory that our simple first-order theory predicts most powerlaw slopes within an accuracy of order $10 \%$. We have also to be aware that our first-order theory assumes that the fractal dimension of the energy release rate is a constant, while the simulated avalanches show a slight trend of increasing fractal dimensions $D$ with size $L$, i.e., $D_{2} \approx L^{0.09}$ for $S=2$, and $D_{3} \approx L^{0.19}$ for $S=3$ (Figs. 7 and 8 , bottom right). This slight trend of $D_{S}(L)$ affects the powerlaw slopes $\alpha_{P}$ and $\alpha_{E}$ to be somewhat flatter for small avalanches than for larger ones, which represents a second-order effect and could be considered in a more refined theory.

\subsection{SOC parameter correlations}

An alternative method of testing our theory is (i) a determination of the power index $\beta$ of correlated parameters $x$ and $y$, i.e., $y \propto x^{\beta}$, by linear regression fits $\log (y) \propto \log \left(y_{0}\right)+\beta \log (x)$; or (ii) by inferring them from the powerlaw slopes $\alpha_{x}$ and $\alpha_{y}$ of their occurrence frequency distributions, i.e., $\beta=\left(\alpha_{x}-1\right) /\left(\alpha_{y}-1\right)$ (see derivation in Sect. 7.1.6 of Aschwanden 2011). The resulting values are listed for both methods in Table 3 (labeled with "Regression" and "Slopes"), for each 1-D, 2-D, and 3-D simulation run of our cellular automaton code. The linear regression fits are shown in the lower halves of Figs. 6-8. Note that truncations occurs for each parameter due to the effect of finite-size systems that have been used in the numerical simulations $(L=128$ and 256 for 1-D; $L=32$ and 64 for 2-D; and $L=16$ and 24 for 3-D lattices). The minimum amount of dissipated energy for a threshold of $B_{\mathrm{c}}=5$ is $E_{\min }=16.7$ (for $S=1$ ), $E_{\min }=20$ (for $S=2$ ), and $E_{\min }=21.4$ (for $S=3$ ), according to Eq. (25). Thus we perform the linear regression fits only in parameter ranges 
M. J. Aschwanden: A statistical fractal-diffusive avalanche model of a slowly-driven self-organized criticality system
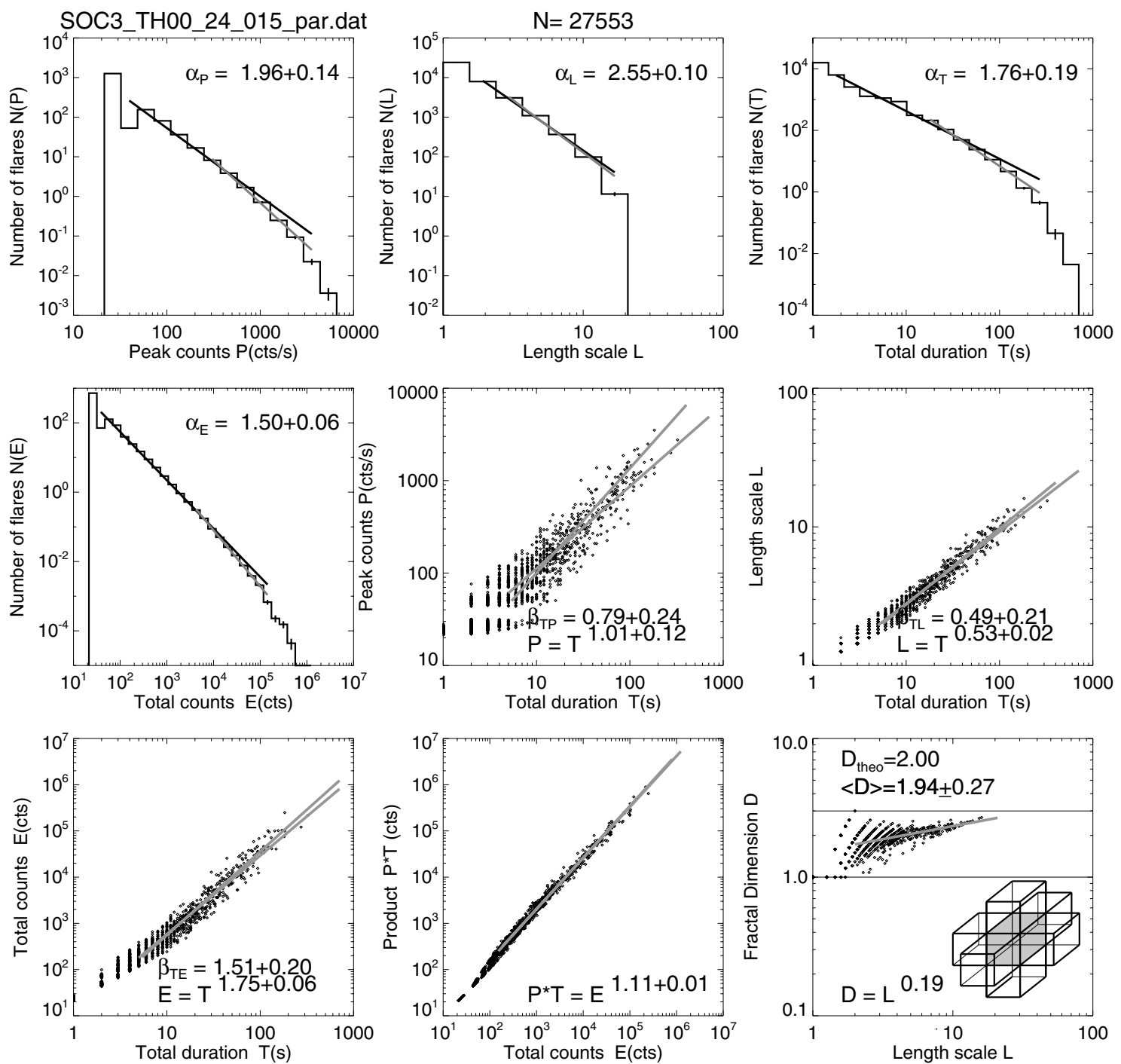

Fig. 8. Cellular automaton simulations with a $N=24^{2} 3$-D lattice produced by a numerical code according to Charbonneau et al. (2001). Representation otherwise similar to Figs. 6 and 7.

that are not too strongly affected by truncation effects, which is at durations $T>5$, peak energy dissipation rate $P>50$, and energies $E>50$. To quantify an error of the linear regression fits, we perform fits of $y(x)$, and with exchanged axes, $x(y)$, and quote the mean and half difference for the two fits.

The correlation $L \propto T^{1 / 2}$ (Eq. (4)) tests our assumption of a diffusive random walk for the propagating avalanche boundaries. For the theoretically expected value of the power in$\operatorname{dex} \beta_{T L}=0.5$ we find $\beta_{T L}=0.53-0.65$ for $1-\mathrm{D}$ avalanches, $\beta_{T L}=0.61-0.76$ for $2-\mathrm{D}$ avalanches, and $\beta_{T L}=0.48-0.53$ for 3 -D avalanches, which corroborates our assumption of a diffusive avalanche expansion.

For the correlation of the peak energy dissipation rate with the duration of an avalanche $P \propto T^{\beta_{T P}}$ we find $\beta_{T P}=0.67-0.73$ for 1-D avalanches (predicted $\beta_{T P}=0.5$ ), $\beta_{T P}=0.91-1.04$ for 2-D avalanches (predicted $\beta_{T P}=1.0$ ), and $\beta_{T P}=0.79-1.02$ for 3-D avalanches (predicted $\beta_{T P}=1.5$ ), which amounts to an agreement of $\approx 30 \%$ for the 1 -D case, $\approx 3 \%$ for the 2 -D case, and $\approx 33 \%$ for the 3 -D case. We suspect that a lot of avalanches are stopped at the boundary, which underestimates the peak energy dissipation rate and thus yields a systematically too high power index $\beta_{T P}$ for the 3-D case.
For the correlation of the total energy with the duration of an avalanche $E \propto T^{\beta_{T E}}$ we find $\beta_{T E}=1.56-1.65$ for $1-\mathrm{D}$ avalanches (predicted $\beta_{T P}=1.5$ ), $\beta_{T E}=1.61-1.82$ for $2-\mathrm{D}$ avalanches (predicted $\beta_{T P}=1.75$ ), and $\beta_{T E}=1.51-1.75$ for 3 $\mathrm{D}$ avalanches (predicted $\beta_{T P}=2.0$ ), which amounts to an agreement of $\approx 7 \%$ for the 1 -D case, $\approx 2 \%$ for the 2 -D case, and $\approx 18 \%$ for the 3-D case.

In summary, we find an overall agreement of order $10 \%$ between theory and numerical simulations for the power indexes of correlated parameters.

\section{Observations of solar flares}

Let us turn now to some astrophysical observations to compare our theory and the results of cellular automaton simulations. $\mathrm{Lu}$ \& Hamilton (1991) applied SOC theory and cellular automaton models for the first time to solar flares. They modeled the flare statistics from hard X-ray counts observed with the HXRBS detectors onboard SMM, which shows a powerlaw distribution extending over 4 orders of magnitude, with a powerlaw slope of $\alpha_{P} \approx 1.8$ for the peak count rate $P$ (Dennis 1985). From their cellular automaton code they obtained powerlaw slopes of $\alpha_{P} \approx 1.8$ for the peak energy dissipation rate $P, \alpha_{T} \approx 1.9$ for 
Table 2. Theoretically predicted and numerically simulated powerlaw slopes of occurrence frequency distributions of cellular automaton models with Euclidean dimension $S=1,2,3$ in the state of self-organized criticality.

\begin{tabular}{|c|c|c|c|}
\hline Reference & $S=1$ & $S=2$ & $S=3$ \\
\hline \multicolumn{4}{|l|}{ Fractal Dimension: $D_{S}$} \\
\hline Theory: $D_{S}=(1+S) / 2$ & 1.00 & 1.50 & 2.00 \\
\hline Simulation $(N=256,64,24)$ & $1.00 \pm 0.00$ & $1.60 \pm 0.17$ & $1.94 \pm 0.27$ \\
\hline Simulation $(N=128,32,16)$ & $1.00 \pm 0.00$ & $1.62 \pm 0.18$ & $1.97 \pm 0.29$ \\
\hline Charbonneau et al. (2001) & & $1.58 \pm 0.02$ & $1.78 \pm 0.01$ \\
\hline McIntosh et al. (2002) & & $1.58 \pm 0.03$ & $1.78 \pm 0.01$ \\
\hline \multicolumn{4}{|l|}{ Length scale powerlaw slope: $\alpha_{L}$} \\
\hline Theory: $\alpha_{L}=S$ & 1.00 & 2.00 & 3.00 \\
\hline Simulation $(N=256,64,24)$ & $0.88 \pm 0.09$ & $2.14 \pm 0.18$ & $2.55 \pm 0.10$ \\
\hline Simulation $(N=128,32,16)$ & $0.89 \pm 0.10$ & $1.96 \pm 0.09$ & $2.58 \pm 0.11$ \\
\hline \multicolumn{4}{|l|}{ Energy powerlaw slope: $\alpha_{E}$} \\
\hline Theory: $\alpha_{E}$ & 1.00 & 1.28 & 1.50 \\
\hline Simulation $(N=256,64,24)$ & $1.06 l \pm 0.04$ & $1.48 \pm 0.03$ & $1.50 \pm 0.06$ \\
\hline Simulation $(N=128,32,16)$ & $1.09 \pm 0.05$ & $1.42 \pm 0.03$ & $1.51 \pm 0.06$ \\
\hline Charbonneau et al. (2001) & & $1.42 \pm 0.01$ & $1.47 \pm 0.02$ \\
\hline McIntosh et al. (2002) & & $1.41 \pm 0.01$ & $1.46 \pm 0.01$ \\
\hline \multicolumn{4}{|c|}{ Peak energy rate powerlaw slope: $\alpha_{P}$} \\
\hline Theory: $\alpha_{P}$ & 1.00 & 1.50 & 1.67 \\
\hline Simulation $(N=256,64,24)$ & $0.94 \pm 0.18$ & $1.85 \pm 0.06$ & $1.96 \pm 0.14$ \\
\hline Simulation $(N=128,32,16)$ & $1.05 \pm 0.10$ & $1.73 \pm 0.08$ & $1.95 \pm 0.13$ \\
\hline Charbonneau et al. (2001) & & $1.72 \pm 0.02$ & $1.90 \pm 0.03$ \\
\hline \multicolumn{4}{|l|}{ Duration powerlaw slope: $\alpha_{T}$} \\
\hline Theory: $\alpha_{T}$ & 1.00 & 1.5 & 2.00 \\
\hline Simulation $(N=256,64,24)$ & $1.17 \pm 0.02$ & $1.77 \pm 0.18$ & $1.76 \pm 0.19$ \\
\hline Simulation $(N=128,32,16)$ & $1.27 \pm 0.15$ & $1.72 \pm 0.10$ & $1.76 \pm 0.18$ \\
\hline Charbonneau et al. (2001) & & $1.71 \pm 0.01$ & $1.74 \pm 0.06$ \\
\hline
\end{tabular}

Table 3. Theoretically predicted and numerically simulated correlations between the length scale $L$, time duration $T$, peak energy dissipation rate $P$, and total energy $E$ for cellular automaton models with Euclidean dimension $S=1,2,3$ in the state of self-organized criticality.

\begin{tabular}{lccc}
\hline \hline Reference & $S=1$ & $S=2$ & $S=3$ \\
\hline Fractal Dimension: $D_{S}$ & & & \\
\hline Diffusive scaling of time with length: $L \propto T^{\beta_{T L}}=T^{1 / 2}$ & & & \\
\hline Theory: $\beta_{T L}$ & $\mathbf{0 . 5 0}$ & $\mathbf{0 . 5 0}$ & $\mathbf{0 . 5 0}$ \\
Slopes $(N=256,64,24)$ & & $0.68 \pm 0.25$ & $0.49 \pm 0.21$ \\
Slopes $(N=128,32,16)$ & $0.53 \pm 0.15$ & $0.76 \pm 0.13$ & $0.48 \pm 0.21$ \\
Regression $(N=256,64,24)$ & $0.65 \pm 0.13$ & $0.61 \pm 0.01$ & $0.53 \pm 0.02$ \\
Regression $(N=128,32,16)$ & & & \\
Correlation of power with duration: $P \propto T^{\beta_{T P}}$ & $\mathbf{0 . 5 0}$ & $\mathbf{1 . 0 0}$ & $\mathbf{1 . 5 0}$ \\
Theory: $\beta_{T P}$ & & $0.91 \pm 0.19$ & $0.79 \pm 0.24$ \\
Slopes $(N=256,64,24)$ & $0.67 \pm 0.20$ & $1.04 \pm 0.12$ & $0.80 \pm 0.22$ \\
Slopes $(N=128,32,16)$ & $0.73 \pm 0.14$ & $1.01 \pm 0.08$ & $1.02 \pm 0.12$ \\
Regression $(N=256,64,24)$ & & & \\
Regression $(N=128,32,16)$ & $\mathbf{1 . 5 0}$ & $\mathbf{1 . 7 5}$ & $\mathbf{2 . 0 0}$ \\
Correlation of energy with duration: $E \propto T^{\beta_{T E}}$ & & $1.61 \pm 0.08$ & $1.51 \pm 0.20$ \\
Theory: $\beta_{T E}$ & & $1.73 \pm 0.10$ & $1.48 \pm 0.19$ \\
Slopes $(N=256,64,24)$ & $1.56 \pm 0.09$ & $1.80 \pm 0.05$ & $1.75 \pm 0.06$ \\
Slopes $(N=128,32,16)$ & $1.65 \pm 0.07$ & $1.82 \pm 0.04$ & $1.75 \pm 0.06$ \\
Regression $(N=256,64,24)$ & & & \\
Regression $(N=128,32,16)$ & &
\end{tabular}

the flare durations $T$, and $\alpha_{E} \approx 1.4$ for the total energies $E$. We re-analyzed the HXRBS/SMM dataset and obtain the values of $\alpha_{P} \approx 1.73 \pm 0.01$ for the power $P, \alpha_{T} \approx 2.29 \pm 0.25$ for the flare durations $T$, and $\alpha_{E} \approx 1.56 \pm 0.04$ for the total energies $E$ (Fig. 9, left). In addition we re-analyzed the BATSE/CGRO flare data set and obtained similar values of $\alpha_{P} \approx 1.71 \pm 0.06$ for the peak energy dissipation rate $P, \alpha_{T} \approx 2.02 \pm 0.28$ for the flare durations $T$, and $\alpha_{E} \approx 1.49 \pm 0.07$ for the total energies $E$ (Fig. 9, right).

Comparing the observed values with the theoretically predicted values of our 3-D fractal-diffusive SOC model (Sect. 2 and Table 1), we find the following ranges regarding the powerlaw slopes of the occurrence frequency distributions: flare durations $\alpha_{T}=2.02-2.29$ (predicted $\alpha_{T}^{\text {theo }}=2.00$ ), peak energy dissipate rate $\alpha_{P}=1.71-1.73$ (predicted $\alpha_{P}^{\text {theo }}=1.67$ ), and total energies $\alpha_{E}=1.49-1.56$ (predicted $\alpha_{E}^{\text {theo }}=1.50$ ), Thus, theory and observations agree within a few percents, which is even better than the agreement of theory with the cellular automaton simulations in the 3D case (probably due to the limited grid size). The powerlaw index of the peak fluxes was found to vary with the solar cycle within a range of $\alpha_{P} \approx 1.6-1.9$ (Crosby et al. 1993; Biesecker 1994; Bai 1993; Aschwanden 2010a), which 

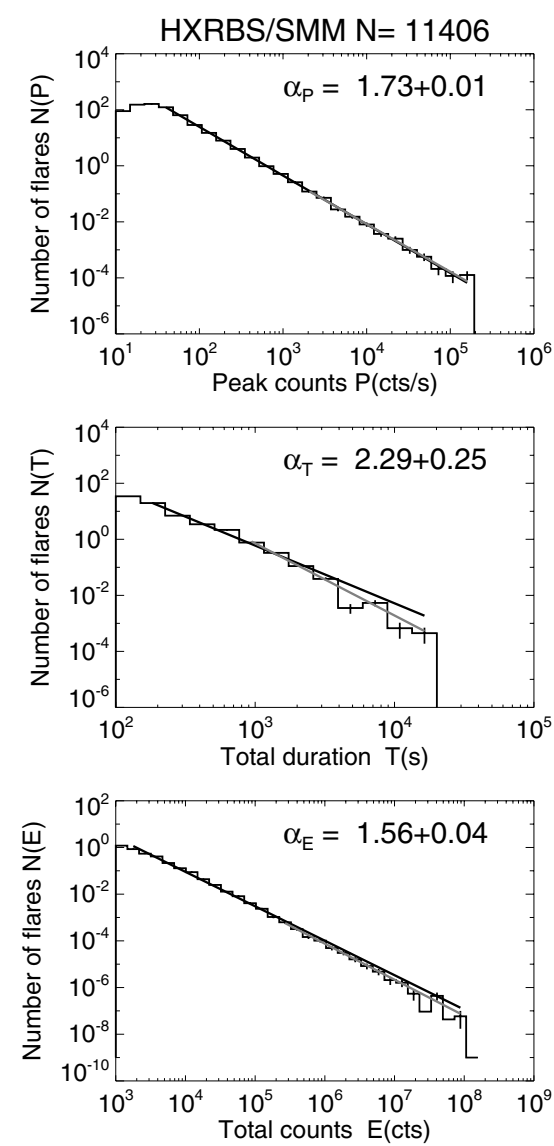
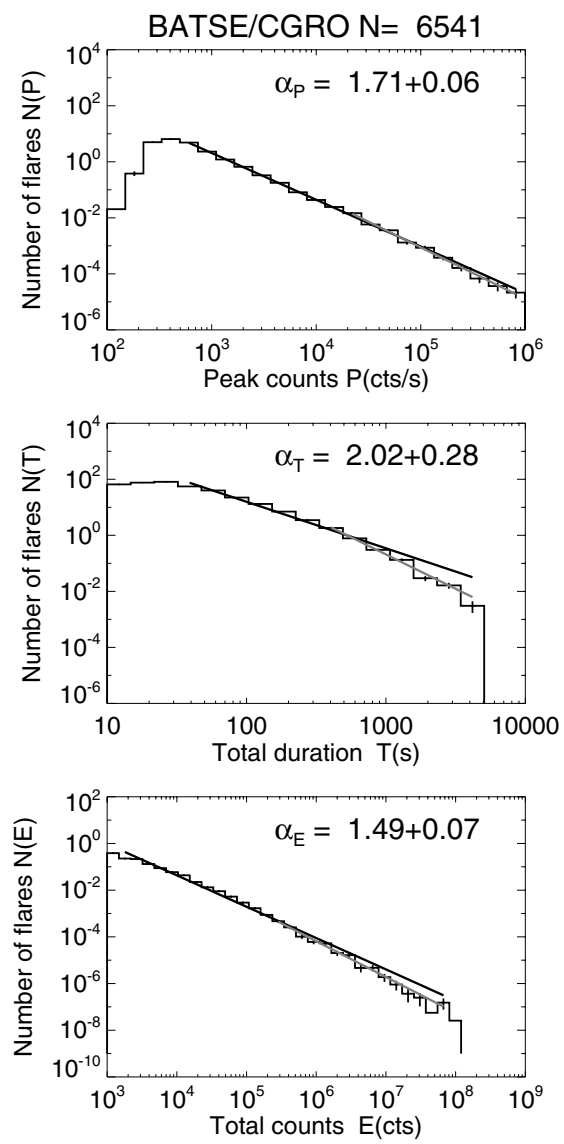

Fig. 9. Occurrence frequency distributions of peak count rates $P$, durations $T$, and total counts $E$ in solar flares observed with HXRBS/SMM during 1980-1989 (left panels) and with BATSE/CGRO during 1991-2000 (right panels) at energies $\geq 25 \mathrm{keV}$ (left). could indicate a variable degree of magnetic complexity that is manifested either in a time-dependent fractal dimension $D_{S}$, or threshold $B_{\mathrm{c}}$ of SOC events (Aschwanden 2010a,b).

Comparing the observed flux time profiles $f(t)$ of the hard $\mathrm{X}$-ray flux of large solar flares, they fluctuate erratically in a similar way as shown for the largest avalanche of cellular automaton simulations (Fig. 3, top left panel), as records with high time-resolution and high sensitivity show, e.g., observed with BATSE/CGRO (Aschwanden et al. 1998). The erratically fluctuating hard X-ray time profiles are generally interpreted in terms of the chromospheric energy dissipation rate of precipitating nonthermal electrons produced in magnetic reconnection processes, similar as we defined the instantaneous energy dissipation rate $f(t)$ (Eq. (8)) in our cellular automaton model, while the associated soft X-ray time profile represents the thermal emission of the heated plasma, which is monotonically increasing during the impulsive flare phase because it represents the time integral of the heating rate according to the Neupert effect, similar as we defined the total dissipated energy $e(t)$ (Eq. (9)) in our cellular automaton model. The positive index in the correlation of the peak energy dissipation rate $P$ with the time duration $T$ in our avalanche model, i.e., $P \propto T^{S / 2}$ (Eq. (10)), thus predicts that the hard X-ray peak flux is statistically the higher the longer a solar flare lasts (which is also known as "big-flare syndrome").

A specific prediction is that in 3-D Euclidean space, regardless of the fractal dimension in the range of $D_{3}=1, \ldots, 3$, the distribution of flare energies is restricted to a relatively small range of $\alpha_{E}^{\text {theo }}=1.40-1.67$, which is consistent with our observations of $\alpha_{E}=1.49-1.56$. The numerical value of the energy powerlaw slope of $\alpha_{E} \approx 1.5$ implies that the total energy of all flares is heavily weighted by the largest flares, while nanoflares contain only an insignificant amount of energy, unless the powerlaw slope is steeper than a critical value of $\alpha_{E}=2$ (Hudson 1991), which is an ongoing argument in the controversy of coronal heating by nanoflares. In order to make nanoflares dominant, a powerlaw slope of $\alpha_{E}>2$ is needed, which contradicts our model, since the maximum value cannot exceed $\alpha_{E \text {,max }}=5 / 3=1.67$, well below the critical limit of $\alpha_{E}=2$.

Interestingly, some cellular automaton simulations have been performed that actually produced significantly steeper powerlaw slopes, say in the order of $\alpha_{E} \approx 3.0$, which seems to contradict our conclusions. One simulation produced a broken powerlaw distribution with a slope of $\alpha_{P}=3.5$ for the smallest events, interpreted as nanoflare regime, while a flatter slope of $\alpha_{P}=1.8$ was found for the larger flares (Vlahos et al. 1995), a difference that is attributed to the anisotropic next-neighbor interactions applied therein. Another study simulated solar flare events as cascades of reconnecting magnetic loops, with the finding of a powerlaw slope of $\alpha_{E}=3.0$ for the released energies (Hughes et al. 2003). Interacting loops with a length scale $L$ are bisected during a reconnection step into two shorter scales $L / 2$, and the released energies are defined in terms of the length scale therein, i.e., $E \propto L$, for which our model predicts indeed an occurrence frequency distribution of $N(E) \propto N(L) \propto L^{-S} \propto L^{-3}$ (Eq. (12)) for an Euclidean dimension $S=3$, so it is fully consistent with our model if their energy definition is adopted. Discrepancies of powerlaw slopes among different studies can indeed often be explained in terms of inconsistent definitions of the energy quantity.

\section{Conclusions}

We developed an analytical theory for the statistical distributions and correlations of observable parameters of SOC events, which 
includes the avalanche length scale $L$, the time duration $T$, the peak $P$ and energy dissipation rate $F$. The basic assumptions of our analytical model, which we call the fractal-diffusive SOC model, are the following:

1. Diffusive expansion of SOC avalanches: the radius $r(t)$ or spatial length scale $L$ of an avalanche grows with time like the average of a diffusive random walk, which predicts a statistical correlation $L \propto T^{1 / 2}$ between the length scale $L$ and time duration $T$ of the avalanche.

2. Fractal energy dissipation rate: the complexity of random next-neighbor interactions in a critical SOC state can be characterized approximately with a fractal geometry. The volume (or area) of the instantaneous energy dissipation rate is assumed to have a fractal dimension $D_{S}$. The predicted statistical correlations are: $F \propto T^{D_{S} / 2}, P \propto T^{S / 2}$, and $E \propto T^{1+D_{S} / 2}$

3. Mean fractal dimension: the mean fractal dimension $D_{S}$ for different Euclidean space dimensions $S=1,2,3$ can be estimated from the arithmetic mean of the minimum dimension for a propagating avalanche, $D_{S, \min } \approx 1$, and the maximum (Euclidean) dimension $D_{S, \max }=S$, which yields $D_{S} \approx(1+S) / 2$.

4. Occurrence frequency distributions: equal probability of avalanches with size $L$ at various spatial locations in a uniform, slowly-driven SOC system predicts a probability distribution of $N(L) \propto L^{-S}$. A direct consequence of this assumption, together with the other assumptions made above, yields a powerlaw function $N(x) \propto x^{-\alpha_{x}}$ for the occurrence frequency distributions of all parameters, which is the hallmark of a SOC system. The predicted powerlaw indices are: $\alpha_{T}=(1+S) / 2, \alpha_{F}=1+(S-1) / D_{S}, \alpha_{P}=2-1 / S$, and $\alpha_{E}=1+(S-1) /\left(D_{S}+2\right)$. Specifically, for applications to 3 -D phenomena, absolute values are predicted for the powerlaw slopes $\alpha_{L}=3, \alpha_{T}=2$, and $\alpha_{P}=1.67$, while a range of $\alpha_{E}=1.4, \ldots, 1.67$ is expected for any fractal dimension in the range of $1 \leq D_{3} \leq 3$.

We have validated our theory by a detailed comparison with a set of SOC simulations using a specific form of a cellular automaton avalanche model (connectivity, stability threshold, redistribution rule, etc.), and found a good agreement between theory and numerical simulations, in the order of $\approx 10 \%$ for the powerlaw slopes $\left(\alpha_{L}, \alpha_{T}, \alpha_{E}, \alpha_{P}\right)$, the power indices of correlated parameters $\left(\beta_{T L}, \beta_{T P}, \beta_{T E}\right)$, and the fractal dimensions $D_{S}$, for all three Euclidean space dimensions $S=1,2,3$. Yet, at its most general level our theory is saying that the self-similarity of energy release statistics in such models is a direct reflection of the fractal nature of avalanches. The SOC flare model recently proposed by Morales \& Charbonneau (2008, 2009) offers an interesting test of this conclusion. Their model, defined on a set of initially parallel magnetic flux strands, contained in a plane and subjected to random sideways deformation, with instability and readjustment occurring when the crossing angle of two flux strands exceeds some threshold angle. This model is thus strongly anisotropic, with pseudo-local stability and redistribution, in the sense that these operators now act on nearestneighbors nodes located along each flux strand, rather than in the immediate spatial vicinity of the unstable sites. This is very different from the isotropic Lu et al. (1993)-type SOC model used here for validation. Yet, the results compiled in Table 2 of Morales \& Charbonneau (2008) for their highest resolution simulations reveal that the theoretical occurrence frequency distributions obtained herein, do hold within the stated uncertainties on the power-law fits. Likewise, the power indices of the correlated parameters also agree with theory within the inferred uncertainties. This provides additional empirical support to our conjecture that the fractal-diffusive SOC model does represent a robust characterization of avalanche energy release in SOC systems in general. However, it should be remembered that the inferred scaling laws are only valid for a slowly-driven SOC system, while alternative SOC systems with time-variable drivers or non-stationary input rates exhibit modified occurrence frequency and waiting time distributions (Charbonneau et al. 2001; Norman et al. 2001), which was also found in solar observations extending over multiple solar cycles (Crosby et al. 1993; Biesecker 1994; Bai 1993; Aschwanden 2010a).

What other predictions can be made from our analytical model? For SOC processes in 3-D space, which is probably the most common application in the real world, the mean fractal dimension is predicted to be $D_{3} \approx 2.0$, which can be tested by measurements of fractal dimensions in observations. The 20 largest solar flares observed with TRACE have been analyzed in this respect and an area fractal dimension of $D_{2}=1.89 \pm 0.05$ was found at the flare peaks, which translates into a value of $D_{3}=2.10 \pm 0.14$ if we use an anisotropic flare arcade model (Aschwanden \& Aschwanden 2008a). The distribution of flare energies is predicted to have a powerlaw slope of $\alpha_{E}=1.50$, which closely matches the observed statistics of solar flare hard $\mathrm{X}$-ray emission $\left(\alpha_{E} \approx 1.49-1.56\right)$. Since this value is undisputably below the critical limit $\alpha=2$ of the energy integral, the total released energy is contained in the largest flares and thus rules out any significant nanoflare heating of the solar corona. Another prediction, that we did not test here with solar flare data, is the diffusive flare size scaling. Straightforward tests could be carried out by gathering statistics of the flare size evolution during individual flares (which are predicted to scale as $x(t) \propto t^{1 / 2}$ ), as well as from the statistics of a large sample of flares, which is predicted to show a correlation $L \propto T^{1 / 2}$. The application of our fractal-diffusive SOC model to solar flares implies that the subsequent triggering of local magnetic reconnection events during a flare occurs as a diffusive random walk. A similar finding of diffusive random walk was also found in the turbulent flows of magnetic bright points in the lanes between photospheric granular convection cells (Lawrence et al. 2001). The spatio-temporal scaling of the diffusive random walk predicts also the size, duration, and energy of the largest flare, which is likely to be constrained by the size $L_{\mathrm{AR}} \propto T_{\max }^{1 / 2}$ of the largest active region.

Acknowledgements. The author thanks Paul Charbonneau for helpful discussions and contributions. This work is partially supported by NASA grant NAG513490 and NASA TRACE contract NAS5-38099. We acknowledge access to solar mission data and flare catalogs from the Solar Data Analysis Center (SDAC) at the NASA Goddard Space Flight Center (GSFC).

\section{References}

Aschwanden, M. J. 2004, Physics of the Solar Corona - An Introduction (New York: Springer/Praxis)

Aschwanden, M. J. 2010a, Sol. Phys., 274, 99

Aschwanden, M. J. 2010b, Sol. Phys., 274, 119

Aschwanden, M. J. 2011, Self-Organized Criticality in Astrophysics - The

Statistics of Nonlinear Processes in the Universe (New York: Springer/Praxis) Aschwanden, M. J., \& Aschwanden, P. D. 2008a, ApJ, 674, 530

Aschwanden, M. J., \& Aschwanden, P. D. 2008b, ApJ, 674, 544

Aschwanden, M. J., Dennis, B. R., \& Benz, A. O. 1998, ApJ, 497, 972 Bai, T. 1993, ApJ, 404, 805

Bak, P., \& Chen, K. 1989, J. Phys. D, 38, 5

Bak, P., Tang, C., \& Wiesenfeld, K. 1987, Phys. Rev. Lett., 59/27, 381

Bak, P., Tang, C., \& Wiesenfeld, K. 1988, Phys. Rev. A, 38/1, 364

Bak, P. 1996, How nature works, Copernicus (New York: Springer-Verlag)

Biesecker, D. A. 1994, Ph.D. Thesis, University of New Hampshire 
M. J. Aschwanden: A statistical fractal-diffusive avalanche model of a slowly-driven self-organized criticality system

Charbonneau, P. S. W., McIntosh, W. W., Liu, H.-L., \& Bogdan, T. J. 2001, Sol. Phys., 203, 321

Crosby, N. B., Aschwanden, M. J., \& Dennis, B. R. 1993, Sol. Phys., 143, 275

Dennis, B. R. 1985, Sol. Phys., 100, 645

Fermi, E. 1949, Phys. Rev. Lett., 75, 1169

Gutenberg, B., \& Richer, C. F. 1954, Seismicity of the Earth and Associated

Phenomena, 2nd edn. (Princeton, NJ: Princeton University Press), 310

Hudson, H. S. 1991, Sol. Phys., 133, 357

Hughes, D., Paczuski, M., Dendy, R. O., Heleander, P., \& McClements, K. G. 2003, Phys. Rev. Lett., 90, 131101

Lawrence, J. K., Cadavid, A. C., Ruzmaikin, A., \& Berger, T. E. 2001, Phys. Rev. Lett., 86, 5894

Litvinenko, Y. E. 1998, A\&A, 339, L57

Liu, H. L., Charbonneau, P., Pouquet, A., Bogdan, T., \& McIntosh, S. 2002, Phys. Rev., 66, 056111

Lu, E. T., \& Hamilton, R. J. 1991, ApJ, 380, L89

Lu, E. T., Hamilton, R. J., McTiernan, J. M., \& Bromund, K. R. 1993, ApJ, 412, 841
Mandelbrot, B. B. 1977, Fractals: form, chance, and dimension, Translation of Les objects fractals (San Francisco: W. H. Freeman)

Mandelbrot, B. B. 1983, The fractal geometry of nature (San Francisco: W. H. Freeman)

Mandelbrot, B. B. 1985, Phys. Scrip., 32, 257

McIntosh, S. W., Charbonneau, P., Bogdan, T. J., Liu, H.-L., \& Norman, J. P. 2002, Phys. Rev. E, 65, 046125

Morales, L., \& Charbonneau, P. 2008, ApJ, 682, 654

Morales, L., \& Charbonneau, P. 2009, ApJ, 698, 1893

Norman, J. P., Charbonneau, P., McIntosh, S. W., \& Liu, H. L. 2001, ApJ, 557, 891

Sornette, D. 2004, Critical phenomena in natural sciences: chaos, fractals, self-organization and disorder: concepts and tools (Heidelberg: Springer), 528

Turcotte, D. L. 1999, Self-organized criticality, Rep. Prog. Phys., 62, 1377

Vlahos, L., Georgoulis, M., Kluiving, R., \& Paschos, P. 1995, A\&A, 299, 897

Willis, J. C., \& Yule, G. U. 1922, Nature, 109, 177 